\title{
Anatomical Variants of "Short Head of Biceps Femoris Muscle" Associated with Common Peroneal Neuropathy in Korean Populations : An MRI Based Study
}

\author{
Jinseo Yang, M.D., ${ }^{1}$ Yongjun Cho, M.D., Ph.D., Jaeho Cho, M.D., ${ }^{2}$ Hyukjai Choi, M.D., Ph.D., Jinpyeong Jeon, M.D., Ph.D., ${ }^{3}$ \\ Sukhyung Kang, M.D., Ph.D.' \\ Departments of Neurosurgery,' Orthopedic Surgery,2 Chuncheon Sacred Heart Hospital, College of Medicine, Hallym University, Chuncheon, Korea \\ Kangwon National University College of Medicine, ${ }^{3}$ Chuncheon, Korea
}

Objective : In Asians, kneeling and squatting are the postures that are most often induce common peroneal neuropathy. However, we could not identify a compatible compression site of the common peroneal nerve (CPN) during hyper-flexion of knees. To evaluate the course of the CPN at the popliteal area related with compressive neuropathy using magnetic resonance imaging (MRI) scans of healthy Koreans.

Methods : 1.5-Tesla knee MRI scans were obtained from enrolled patients and were retrospectively reviewed. The normal populations were divided into two groups according to the anatomical course of the CPN. Type I included subjects with the CPN situated superficial to the lateral gastocnemius muscle (LGCM). Type II included subjects with the CPN between the short head of biceps femoris muscle (SHBFM) and the LGCM. We calculated the thickness of the SHBFM and posterior elongation of this muscle, and the LGCM at the level of femoral condyles. In type II, the length of popliteal tunnel where the CPN passes was measured.

Results : The 93 normal subjects were included in this study. The CPN passed through the "popliteal tunnel" formed between the SHBFM and the LGCM in 36 subjects (38.7\% type II). The thicknesses of SHBFM and posterior portions of this muscle were statistically significantly increased in type II subjects. The LGCM thickness was comparable in both groups. In $78.8 \%$ of the "popliteal tunnel", a length of $21 \mathrm{~mm}$ to $<40 \mathrm{~mm}$ was measured.

Conclusion : In Korean population, the course of the CPN through the "popliteal tunnel" was about $40 \%$, which is higher than the Western results. This anatomical characteristic may be helpful for understanding the mechanism of the CPNe by posture.

Key Words : Peroneal nerve · Fibular nerve · Peroneal neuropathies · Nerve compression syndromes · Entrapment neuropathy.

\section{INTRODUCTION}

Posture-induced common peroneal neuropathy $(\mathrm{CPNe})$ is a clinical presentation caused by a prolonged body position that leads to acute foot drop with sensory disturbances of the anterior or lateral compartments of lower leg. We previously reported the clinical characteristics of posture-induced CPNe and summarized eight helpful diagnostic points for differen-

- Received : January 23, 2018 •Revised : March 14, 2018 •Accepted : March 29, 2018

- Address for reprints : Yongjun Cho, M.D., Ph.D.

Department of Neurosurgery, Chuncheon Sacred Heart Hospital, College of Medicine, Hallym University, 77 Sakju-ro, Chuncheon 24253, Korea Tel : +82-33-240-5171, Fax : +82-50-7084-4867, E-mail : nssur771@hallym.or.kr

This is an Open Access article distributed under the terms of the Creative Commons Attribution Non-Commercial License (http://creativecommons.org/licenses/by-nc/4.0) which permits unrestricted non-commercial use, distribution, and reproduction in any medium, provided the original work is properly cited. 
tial diagnosis ${ }^{11)}$. In Asians, kneeling and squatting are the postures that are most often induce CPNe. However, we could not identify a compatible compression site of the common peroneal nerve (CPN) during hyper-flexion of knees, except for the fibrous-osseous band (fibular tunnel), which was a stretching or direct compression site of the nerve during prolonged and habitual leg crossing and lying down. Masakado et al. ${ }^{6}$ described this tunnel as a compression site of the CPN during hyper-flexion of knee posture, but could not explain the detailed mechanism. Though CPNe is caused by various conditions, the etiology of idiopathic or posture-induced neuropathy remains unclear ${ }^{1,2,5,6,11)}$.

Masakado et al. ${ }^{6)}$ investigated the course of the CPN between popliteal muscles, including the short head of biceps femoris muscle (SHBFM) and the lateral gastrocnemius muscle (LGCM) using T1-weighted magnetic resonance images $(\mathrm{MRI})^{8)}$. They proposed the possibility of anatomical variations on these muscles related to the CPNe by entrapment or compression. We hypothesized that the popliteal fossa is an anatomical region where the CPN could be compressed, rather than the fibular tunnel, and assumed the "popliteal tunnel" formed between the SHBFM and the LGCM could compress the CPN during knee flexion posture.

Presently, we evaluated the course of the CPN at the popliteal area related with compressive neuropathy. We also investigated the incidence of anatomical variation associated with CPN and the morphological characteristics on the basis of comprehensive MRI scans of healthy Koreans.

\section{MATERIALS AND METHODS}

From 2014 January to 2016 December, 1.5-Tesla knee MRI scans were obtained from 849 patients using a Gyroscan Intera machine (Philips Medical Systems, Eindhoven, The Netherlands). These images were retrospectively reviewed. We excluded 756 patients with traumatic lesions (fracture, dislocation, ligament injury, and contusion), post-surgery (total knee arthroplasty and arthroscopic guided procedures), and tumors (cystic and solid) after searching medical records. The remaining 93 normal subjects were included in the study. The subjects were divided into two groups according to the anatomical course of the CPN. Type I included subjects with the CPN situated superficial to the LGCM. Type II included sub- jects with the CPN between the SHBFM and the LGCM. All measured parameters were evaluated on conventional T1weighted axial images (TR, 500-800 ms; TE, 10-20 ms). We calculated the thickness of the SHBFM and posterior elongation of this muscle, and the LGCM at the level of femoral condyles (Fig. 1). In the type II group, the length of popliteal tunnel where the CPN passes was measured on coronal T2weighted Dixon MRI images of knee joints. The thickness of the SHBFM and LGCM, which each comprised popliteal fossa, was determined in each group. All measurements were reviewed independently by a neurosurgeon and an orthopedic surgeon. This study involves no prospectively collected data so there is no access to patients or opportunity to seek informed consent and was performed in accordance with our institutional guidelines that comply with all international laws and policies. This study was performed in accordance with Hallym University College of Medicine guidelines (IRB No.2012-71) that comply with all international laws and policies.

\section{Statistical analyses}

Inter- and intraobserver reliabilities were obtained for all radiographic parameters using the intraclass correlation coef-

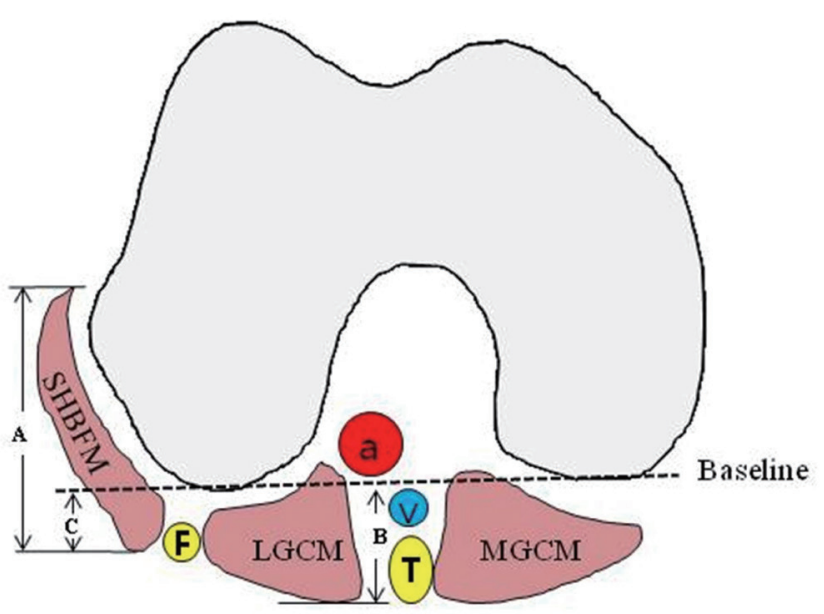

Fig. 1. Schematic description of the measurement method on the right knee. The baseline connected the two posterior apexes of the femoral condyle. The muscle thicknesses that were the vertical length of the SHBFM (A), length and maximum lengths from baseline of the LGCM (B), and the vertical and maximum length from baseline of the posterior elongated length of the SHBFM (C). a : popliteal artery, v : popliteal vein, $\mathrm{T}$ : tibial nerve, $\mathrm{F}$ : common peroneal nerve, SHBFM : short head of biceps femoris muscle, LGCM : lateral gastrocnemius muscle, MGCM : medial gastrocnemius muscle. 
ficient (ICC). As defined ${ }^{4}$, ICCs of $0.81-1.00,0.61-0.80,0.41-$ $0.60,0.21-0.40$, and $0.00-0.20$ were interpreted as excellent, good, moderate, fair, and poor, respectively. After identifying the normal distribution of baseline characteristics between type I and type II, the Mann-Whitney test and Fisher's exact test were used to compare baseline characteristics and radiologic measurements such as thicknesses and ratios of examined muscles. The null hypothesis of no difference was rejected at $p<0.05$. Data were analyzed using the SPSS version 20.0 for Windows (SPSS, Inc., Chicago, IL, USA).

\section{RESULTS}

\section{Baseline characteristics of type I and type II}

Analysis of the CPN course on conventional T1-weighted axial images as described above at the level of femoral condyles revealed that the CPN separated from the sciatic nerve descended along the medial side of the SHBFM on the popliteal fossa and passed through the popliteal tunnel formed between the SHBFM and the LGCM in 36 subjects (38.7\% type II). In 57 subjects (61.3\% type I) the CPN pathway traveled superficially without passing through the popliteal tunnel. The overall median age of the enrolled subjects was 42 years (mean, 40.2 years; range, $10-73$ years). The median age of the type I and type II group was 45 years (mean, 41.3 years; range, 11-73 years) and 38 years (mean, 38.6 years; range, 10-73 years), respectively. More than $50 \%$ of patients $40-60$ years of age in both groups and showed a normal age distribution $(p=0.446)$. Overall, male subjects were more common than females (34 males and 23 females in type I; 23 males and 13 females in type II). The groups were statistically similar significant $(p=0.684)$.

\section{Comparisons of measured thickness in each type}

The SHBFM is located at the distal and lateral side of the popliteal fossa, and the CPN typically runs along the medial side of this muscle. The median thickness was $30.2 \mathrm{~mm}$ (mean, $31.0 \mathrm{~mm}$; range, $19.8-45.6 \mathrm{~mm}$ ) in the type I group and $34.6 \mathrm{~mm}$ (mean, $35.9 \mathrm{~mm}$; range, $21.8-53.0 \mathrm{~mm}$ ) in the type II group. The muscle was significantly thicker in type II subjects (ICC, 0.82; $p=0.005$ ) (Fig. 2A).

The LGCM is located on the bottom of the popliteal fossa and the CPN usually runs to the superior surface of the mus- cle. The median thickness was $18.9 \mathrm{~mm}$ (mean, $20.0 \mathrm{~mm}$; range, $9.6-34.0 \mathrm{~mm}$ ) in type I subjects and $20.3 \mathrm{~mm}$ (mean, $20.2 \mathrm{~mm}$; range, $9.2-31.9 \mathrm{~mm}$ ) in type II subjects. The muscle thickness was comparable in both groups (ICC, $0.86 ; p=0.508$ ) (Fig. 2B).

From the baseline, the median lengths of the SHBFM muscle are $9.0 \mathrm{~mm}$ (mean, $9.3 \mathrm{~mm}$; range, $1.0-19.8 \mathrm{~mm}$ ) in type I subjects and $15.7 \mathrm{~mm}$ (mean, $16.2 \mathrm{~mm}$; range, $6.0-30.4 \mathrm{~mm}$ ) in type II subjects. The posterior portion of this muscle was statistically significantly increased in type II subjects (ICC, $0.74 ; p=0.005$ ) (Fig. 2C).

The ratio of the lengths of the two short head of the biceps femoris and lateral gastrocnemius muscles involved in the course of CPN was calculated in each type. The median ratios were 1.8 (mean, 1.9; range, 1.2-2.8) in type I subjects and 2.1 (mean, 2.2; range, 1.4-3.3) in type II subjects. The difference was significant ( $p=0.005$ ) (Fig. 2D).

\section{Frequencies of popliteal tunnel length in type II (Table 1)}

Almost 54\% of subjects in type II had a tunnel length from 21-30 mm, and there was no subject with tunnel below 10 $\mathrm{mm}$. Over $27 \%$ of subjects had a tunnel length exceeding 31 $\mathrm{mm}$ and maximum length was $52 \mathrm{~mm}$. This subject with maximum tunnel length had $41.1 \mathrm{~mm}$ on the SHBFM, 18.7 $\mathrm{mm}$ on the LGCM, and $23.7 \mathrm{~mm}$ on posterior elongated SHBFM. These outcomes were more than the average, except LGCM length.

\section{Case illustration}

A 52-year-old male farmer presented with right ankle drop. Two days prior to presentation, he had experienced sensory disturbance on right lateral side of lower leg, followed by ankle dorsiflexor muscle weakness. On admission, neurological examination revealed right ankle dorsiflexion (G2/5), big toe dorsiflexion (Gr3/5), ankle eversion (Gr3/5), ankle inversion (G5/5), and ankle plantarflexion (G4/5) with decreased sensation on lateral compartment of lower leg. Conventional knee MRI demonstrated the CPN ran between the SHBFM and the LGCM at the level of femoral condyle, which was classified as type II. Significantly high signal changes on the SHBFM and the LGCM and muscles of anterolateral compartment were observed. The changes were compatible with his clinical presentation (Fig. 3). An electromyography examination per- 

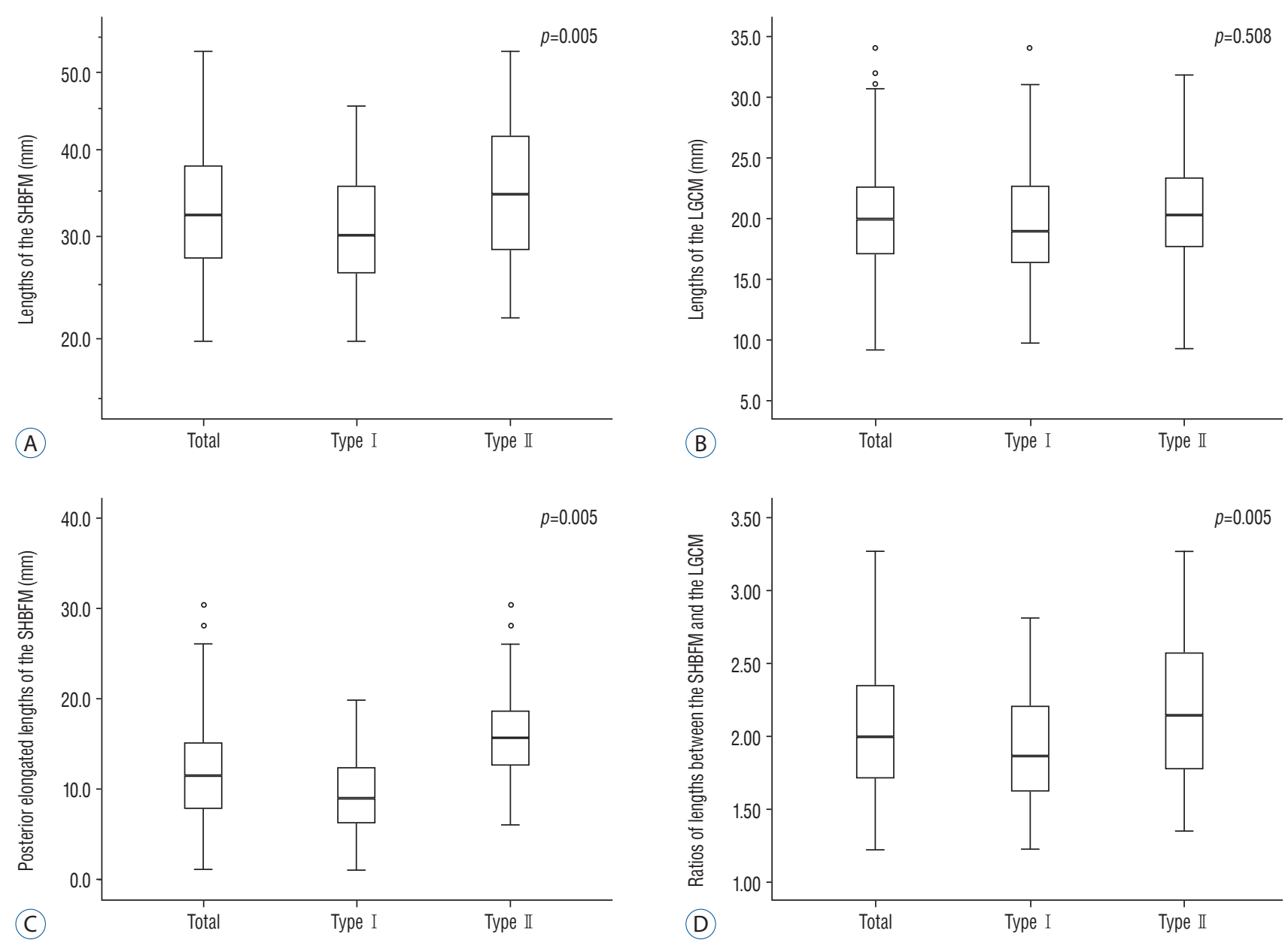

Fig. 2. Comparison of four measured thicknesses in each type. A : Comparison of measurement outcome for length of the short head of biceps femoris muscle (SHBFM) in each type. B : Comparison of measurement outcome for length of the lateral gastrocnemius muscle (LGCM) in each type. $C$ : Comparison of measurement outcome for posterior elongated length of the SHBFM in each type. D : Ratio outcomes of lengths between the SHBFM and the LGCM in each type.

Table 1. Frequencies of the popliteal tunnel length in type II

\begin{tabular}{lc}
\hline Tunnel length $(\mathbf{m m})$ & No. of subjects \\
\hline$\leq 10$ & $0(0)$ \\
11 to 20 & $7(19.4)$ \\
21 to 30 & $19(53.8)$ \\
31 to 40 & $9(25)$ \\
$\geq 41$ & $1(2.8)$ \\
Total & $36(100)$ \\
\hline
\end{tabular}

Values are presented as number (\%)

formed 17 days after the initial symptom of sensory disturbance showed signs of abnormal spontaneous activity in the right tibialis anterior muscle, peroneus longus muscle, and extensor digitorum brevis muscle with diminished recruitment
(Table 2). He was diagnosed with incomplete fibular neuropathy and was managed with physiotherapy. A follow-up 1.5 months later, his affected muscles were improved to over grade 4 .

\section{DISCUSSION}

The common peroneal nerve branches from the sciatic nerve and descends obliquely along the lateral side of popliteal fossa to the head of the fibula, close to the medial margin of the biceps femoris muscle laying between the tendon of the biceps femoris and lateral head of the gastrocnemius muscle, and then winds around the neck of the fibula ${ }^{1,2,5,6}$. MRI findings have demonstrated that the common peroneal nerve de- 

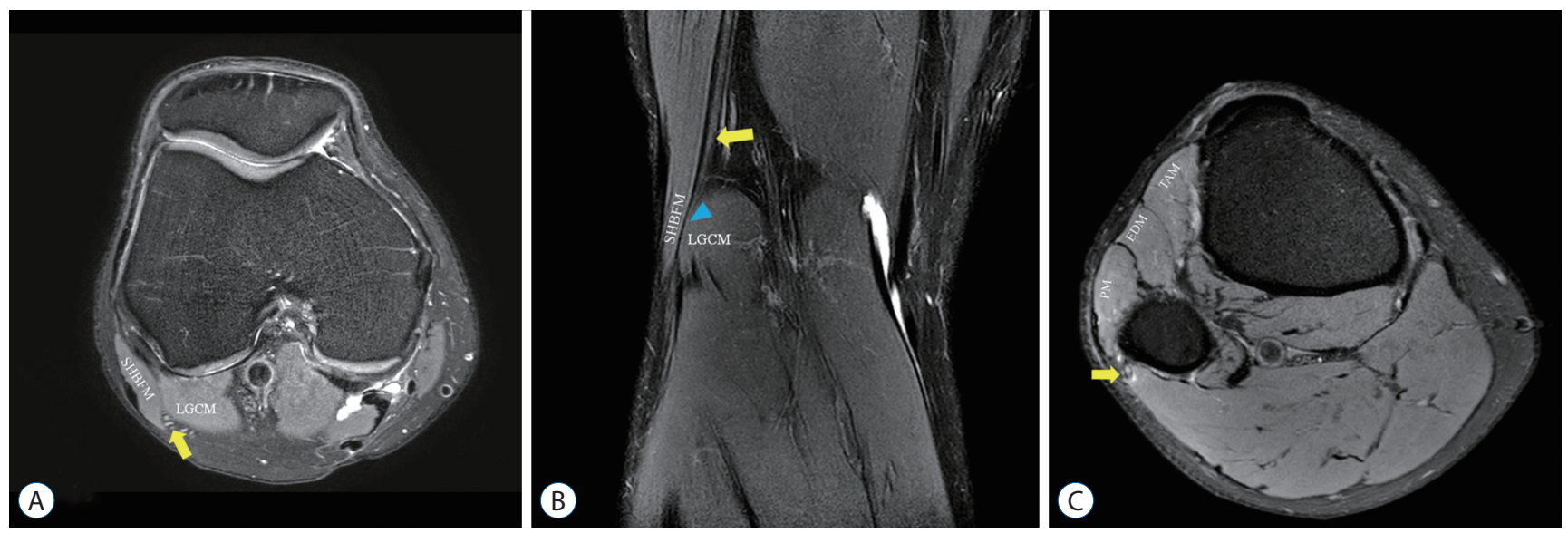

Fig. 3. Conventional 1.5T knee MRI findings. A : Axial proton density fat-saturated MRI (TR, $5100 \mathrm{~ms} ; T E, 30 \mathrm{~ms}$ ) at the level of femoral condyle was done to image the suspected course of the CPN (yellow arrow) between the SHBFM and the LGCM. B : Coronal mDIXON T2-weighted MRI (TE, 2500 ms; TE, 80 ms) revealed the CPN (yellow arrow) descending along the medial side of the SHBFM, passing through the popliteal tunnel (blue arrowhead), and traveling down to the lateral side of the fibula. The length of this tunnel was $24 \mathrm{~mm}$. C : Axial fat-saturated T2-weighted MRI (TR, $3500 \mathrm{~ms}$; TE, $65 \mathrm{~ms}$ ) at the level of proximal entrance to fibular head showed the swollen CPN (yellow arrow) and demonstrated edematous high signal changes within muscles of antero-lateral compartment of lower extremity. MRI : magnetic resonance imaging, CPN : common peroneal nerve, mDIXON : modified DIXON, SHBFM : short head of biceps femoris muscle, LGCM : lateral gastrocnemius muscle, MGCM : medial gastrocnemius muscle, PM : peroneus muscle, EDM : extensor digitorum muscle group, TAM : tibialis anterior muscle.

Table 2. Initial electromyography in the right lower extremity

\begin{tabular}{|c|c|c|c|c|c|}
\hline \multirow{2}{*}{ Muscles (right side) } & \multicolumn{3}{|c|}{ Spontaneous activity } & \multirow{2}{*}{ MUAP } & \multirow{2}{*}{ Recruitmen } \\
\hline & IA & Fib & PSW & & \\
\hline \multicolumn{6}{|l|}{ PSP } \\
\hline L3-L4 & $\mathrm{N}$ & None & None & $\mathrm{N}$ & \\
\hline L4-L5 & $\mathrm{N}$ & None & None & $\mathrm{N}$ & \\
\hline L5-S1 & N & None & None & N & \\
\hline Tibialis anterior & Increased & $1+$ & $2+$ & N & $P$ \\
\hline Peroneus longus & Increased & $1+$ & $2+$ & $\mathrm{N}$ & P \\
\hline Extensor digitorum brevis & Increased & $1+$ & $3+$ & N & $P$ \\
\hline Gastrocnemius & N & N & None & N & $\mathrm{P}-\mathrm{C}$ \\
\hline Vastus medialis & N & $\mathrm{N}$ & None & N & $\mathrm{P}-\mathrm{C}$ \\
\hline
\end{tabular}

IA : insertion activity, Fib : fibrillation, PSW : positive sharp wave, MUAP : motor unit action potential, PSP : paraspinal, N : normal response, P : partial, P-C : partial to complete

scends obliquely along the lateral side of the popliteal fossa, posterior to the short head of biceps femoris muscle, and then laterally and superficially to the lateral head of the gastrocnemius muscle. More inferiorly, it winds around the neck of the fibula (fibular tunnel), enters the anterior and lateral muscle compartments of the leg, and divides into the superficial and deep peroneal nerves ${ }^{3,5)}$. Anatomical variations of peroneal nerve occur in humans. Deutsch et al. ${ }^{2)}$ studied the relevant regional anatomy of common peroneal nerve and reported three distinct patterns of division in the common peroneal nerve with respect to the deep and superficial branches: division distal to fibular neck (81.4\%), division proximal to joint line $(10 \%)$, division distal to joint line and proximal to fibular neck $(8.6 \%)$. The authors demonstrated the potential risk for nerve injury during repair of lateral meniscus tears according to these types of variations ${ }^{2}$. In another cadaveric study, Arora et al. ${ }^{1 .}$ also reported an anormalous variation of the common peroneal nerve at the level of the middle of popliteal fossa and emphasized its clinical importance. Nevertheless, these anatomical variations of peroneal nerve could not fully explain all 
possible causes or mechanisms of the CPNe.

Previously, we reported the clinical characteristics of peroneal nerve palsy that developed after maintaining a certain posture for a long time and suggested that this entrapment syndrome was related to the seating habits of Asians ${ }^{11)}$. Posture-induced CPNe with neurologic deficit can be caused by impaired blood supply to the nerve due to direct compression or stretching injury after prolonged posture ${ }^{7)}$. In some cases of external direct compression to the CPN while lying down in the lateral decubitus position, the fibular tunnel or the fibular head seemed to be an entrapment of the compression site, leading to the CPNe. Postures that commonly induce CPNe are squatting (54\%), sitting cross-legged (25\%) and are affected by maintaining the same posture for an average of 124.2 minutes ${ }^{11)}$. Masakado et al. ${ }^{6}$ mentioned that habitual leg crossing may repetitively injure the peroneal nerve at the fibular neck and, similarly, repetitive stretching from squatting has also been associated with CPNe. However, we could not fully understand the mechanism of the CPNe in these postures and also could not find related literature. Since these postures involve flexion of knees with hip flexion, the popliteal fossa would be the most affected area, where the peroneal nerve could be injured rather than the fibular tunnel. Therefore, we suggest that kneeling or prolonged squatting positions may affect the CPN on the lateral side of the popliteal fossa composed of the SHBFM and the LGCM.

The anatomical features or variations of the SHBFM were first reported by Vieira et al. ${ }^{8)}$. They investigated the correlation of the CPN with the SHBFM at level II of the $\mathrm{CPN}^{5}$, and from the apex of popliteal triangle to proximal fibular tunnel by $1.5 \mathrm{~T} \mathrm{MRI}$. The authors described the presence of this variation as a narrow fatty tunnel formed between the SHBFM and the LGCM as a possible entrapment area. The tunnels formed by the posterior elongation of the SHBFM at the position where the fat pad of LGCM should be existed consisted of floor by the LGCM and roof by the SHBFM and these two muscles are anatomical landmarks related to the pathways of the CPN in the popliteal area. For this reason we use this term, popliteal tunnel, to describe the anatomical variations located in the level II of the CPN course, though this is not officially used. The study had a low incidence (23\%) of this variation compared to our study (almost 40\%). The frequency of length of tunnel also differed from our study. In $45.8 \%$ of patients, a length $<20 \mathrm{~mm}$ was confirmed, while in our study, $19.4 \%$ of subjects showed a similar length. In 50\% of subjects, a length of $21 \mathrm{~mm}$ to $<40 \mathrm{~mm}$ was measured, while $78.8 \%$ of subjects displayed this range in our study.

We found that the proportion of the SHBFM and the LGCM was higher in type II, and the length of the LGCM was not statistically different between two types. Therefore, it could be assumed that the existence of the popliteal tunnel is related to the development or thickness of the SHBFM. The SHBMF is the most lateral component of the hamstring muscle on the posterior thigh. As its name implies, it has two parts. One is the long head, which arises from the tuberosity of the ischium and the other is the short head that arises from the lateral lip of the linea aspera. Both heads of the biceps femoris muscle perform knee flexion and the long head is involved in hip extension. Visser et al. ${ }^{9)}$ studied the effect of changing the hip angle on the length of biceps femoris muscle and reported the change of angulation of hip joint has much larger elongation effect on the length of biceps femoris than the change of knee angulation by cadaveric experiment. In Asian culture, repetitive squatting position and habitual legcrossing feature knee flexion with change of hip angulation. Athletes also use the biceps femoris muscle frequently ${ }^{10)}$. We suggest that the culture of sitting on the floor and an environment (like sports) that features repetitive squatting position are important in the induction of the development of the biceps femoris muscle. In circumstances requiring maintained motion of knee flexion, patients with popliteal tunnel because of development of biceps femoris muscle may be easily exposed to opportunities for the CPN to be squeezed. We assume that racial difference and sedentary or leg-crossing cultures may be the reasons for the existence of popliteal tunnel, but detailed prospective analyses of the physical characteristic, occupations, and life styles are needed to prove why the popliteal tunnel exists.

Study limitations include potential biases due to the retrospective MRI study design and single institution involvement, small number of populations. The results of our analysis cannot be generalized to all populations.

\section{CONCLUSION}

In normal Korean population, the course of the CPN through the "popliteal tunnel" formed between the SHBFM 
and the LGCM was about $40 \%$, which is higher than the Western results. This anatomical characteristic may be helpful for understanding the mechanism of the CPNe by posture.

\section{CONFLICTS OF INTEREST}

No potential conflict of interest relevant to this article was reported.

\section{INFORMED CONSENT}

This type of study does not require informed consent.

\section{- Acknowledgements}

This research was supported by Hallym University Research Fund 2016-48 (HURF 2016-48) and BioGreen21 Program (PJ013139012018) of Rural Development Administration.

\section{References}

1. Arora AK, Verma $\mathrm{P}, \mathrm{Abrol} \mathrm{S}$ : An anomalous variation in the division pattern of the common peroneal nerve. Int J Appl Basic Med Res 1 :
118-119, 2011

2. Deutsch A, Wyzykowski RJ, Victoroff BN : Evaluation of the anatomy of the common peroneal nerve. Defining nerve-at-risk in arthroscopically assisted lateral meniscus repair. Am J Sports Med 27 : 10-15, 1999

3. Donovan A, Rosenberg ZS, Cavalcanti CF : MR imaging of entrapment neuropathies of the lower extremity. Part 2. The knee, leg, ankle, and foot. Radiographics 30 : 1001-1019, 2010

4. Landis JR, Koch GG : The measurement of observer agreement for categorical data. Biometrics 33 : 159-174, 1977

5. Loredo R, Hodler J, Pedowitz R, Yeh LR, Trudell D, Resnick D : MRI of the common peroneal nerve: normal anatomy and evaluation of masses associated with nerve entrapment. J Comput Assist Tomogr 22 : 925 931, 1998

6. Masakado Y, Kawakami M, Suzuki K, Abe L, Ota T, Kimura A : Clinical neurophysiology in the diagnosis of peroneal nerve palsy. Keio J Med 57 : 84-89, 2008

7. Rempel DM, Diao E : Entrapment neuropathies: pathophysiology and pathogenesis. J Electromyogr Kinesiol 14 : 71-75, 2004

8. Vieira RL, Rosenberg ZS, Kiprovski K : MRI of the distal biceps femoris muscle: normal anatomy, variants, and association with common peroneal entrapment neuropathy. AJR Am J Roentgenol 189 : 549-555, 2007

9. Visser JJ, Hoogkamer JE, Bobbert MF, Huijing PA : Length and moment arm of human leg muscles as a function of knee and hip-joint angles. Eur J Appl Physiol Occup Physiol 61 : 453-460, 1990

10. Watemberg $N$, Amsel $S$, Sadeh $M$, Lerman-Sagie $T$ : Common peroneal neuropathy due to surfing. J Child Neurol 15 : 420-421, 2000

11. Yu JK, Yang JS, Kang SH, Cho YJ : Clinical characteristics of peroneal nerve palsy by posture. J Korean Neurosurg Soc 53 : 269-273, 2013 